Open Access

\title{
Construction of BAC contig maps of homoeologous chromosomes A12 and D12 of Gossypium hirsutum L. acc. TM-1
}

Yanhui Lv, Dan Ma, Wenhua Liang, Yuanda Lv, Wangzhen Guo, Yan Hu* and Tianzhen Zhang*

\begin{abstract}
Background: The Gossypium hirsutum homoeologous chromosome 12 encodes important genes that contribute to fiber fuzz, lethality, gland development and male sterility. In this study a physical map of the cotton TM-1 chromosome 12 was constructed. A number of large-insert cotton genome libraries are available, and genome-wide physical mapping using large insert segments combined with bacterial cloning is a thriving area of genome research. However, sequencing of the cotton genome is difficult due to sequence repeats and homoeologous regions. In order to effectively distinguish the homologous segments, a new method for adjusting the parameters of the FPC software was applied for contig map construction.

Results: All available markers on chromosomes A12 and D12 were used to screen the TM-1 BAC library by PCR. A total of 775 clones (387 for A12, 388 for D12) were obtained using Hind III fingerprinting and used for construction of the contig map. Seven pairs of SSR markers located on A12 and D12 were chosen for contig analysis. Following optimization of the tolerance (10) and cutoff (1e-12) parameters, combining all clones from A12 and D12 produced two separate contigs.
\end{abstract}

Conclusions: The BAC contig map of chromosomes A12 and D12 was constructed and FPC software parameters were optimized for analysis. The resulting approach is a powerful platform for genome-wide and evolutionary research on cotton.

Keywords: Contig, BAC, Fingerprint, Homoeologous chromosome, Cotton, Genomics

\section{Background}

BACs (bacterial artificial chromosomes) are important resources for map-based cloning and large-scale sequencing of complex genomes due to their ability to stably maintain large DNA fragments that facilitate easy manipulation [1,2]. BAC clones can be arranged into contigs which are contiguous, gap-free overlapping clones $[3,4]$ which can be used to identify the minimum tilling path fingerprinted contigs required for further gene cloning. BAC libraries have been constructed from various different cotton species, including Maxxa [5], Suyuan7235 [6], Zhongmiansuo 12 [7], 0-613-2R [8], and Pima 90-53 [9]. These libraries can be used to construct a high quality physical map of the cotton genome.

\footnotetext{
* Correspondence: njauhuyan@njau.edu.cn; cotton@njau.edu.cn State Key Laboratory of Crop Genetics \& Germplasm Enhancement, Hybrid Cotton R \& D Engineering Research Center, MOE, Nanjing Agricultural University, Nanjing 210095, China
}

Cotton is the source of the world most important plant-derived fiber, and is also an important oilseed crop, as well as a model species for the study of plant polyploidy, cellulose biosynthesis and cell wall biogenesis [10]. The genus Gossypium consists of over 50 species, including $40-45$ diploids $(2 \mathrm{n}=2 \times=26)$ and five allotetraploids $(2 n=4 \times=52)$ [11] that were formed from separate $\mathrm{A}$ and $\mathrm{D}$ genomes through polyploidization 1-2 million years ago [12-14]. The tetraploid cotton genome contains 13 pairs of homologous chromosomes [15-17] which have been incorporated into specific BAC clones, and a new chromosome nomenclature for tetraploid cotton has been proposed [18]. A12 and D12 chromosomes in allotetraploid cotton are among the most important pairs of homoeologous chromosomes, and include the alleles $\left(N_{1}, n_{2}\right)$ at naked seeds [19], $L e_{1}, L e_{2}$ at hybrid lethality $[20,21], g l_{2}, g l_{3}$ at gossypol glanding, $n e_{1}, n e_{2}$ at mecariless, $B w_{1}, B w_{2}$ at withering bract, $m s_{8}, m s_{9}$ at male 
sterile [22-26]. For map-based cloning and wholegenome sequencing of tetraploid cotton, a BAC library for G. hirsutum acc. TM-1 has been constructed [8, 27, 28]. Furthermore, a high-density gene-rich genetic map containing 2247 loci and covering 3540.4-cM, with an average inter-marker distance of 1.58-cM, has been constructed from the $\mathrm{BC}_{1}(\mathrm{TM}-1 \times$ Hai7124) cross [29-34].

In this study, to facilitate construction of BAC contig maps for the G. hirsutum L. Acc. TM-1 homoeologous chromosomes A12 and D12, the molecular markers identified in the aforementioned genetic map were used to screen the BAC library. BACs containing genetic markers that produced characteristic fingerprints when digested with the restriction enzyme Hind III were used to build contigs using the FPC software. A BAC fingerprint-based contig map of both A12 and D12 was successfully constructed by adjusting the tolerance and cutoff parameters in FPC. This approach will facilitate future map-based cloning of important cotton genes and expand our understanding of the relationships between the genetic and physical maps of A12 and D12.

\section{Results}

Identifying BAC clones containing genes from chromosomes A12 and D12

BAC library screening was based on the published linkage map [33], and a total of 101 and 124 SSR markers from tetraploid cotton homoeologous chromosomes A12 and D12, respectively, were selected for screening the TM-1 library by a PCR-based method [27]. The PCR products were abundant and well-resolved, and positive BAC clones were identified by the SSR marker. In total, 775 positive BAC clones were screened out using the SSR markers, and each marker identified and average of five clones.

A12 and D12 form a pair of homoeologous chromosomes, and previous research confirmed the presence of large homologous segments shared between them. BACs can usually be distinguished using SSR markers based on polymorphic loci. For example, the SSR marker dPL0240 produced two fragments in G. barbadense cv. Hai7124 and two in G. hirsutum acc. TM-1 (Fig. 1). One was a polymorphic locus, dPL0240_160 in Hai7124 and dPL0240_155 in TM-1, which mapped to chromosome 12. Eight BACs amplified using dPL0240 produced identical bands in TM-1. These BACs were mapped to A12, again based on the polymorphic marker. The other fragment generated by the SSR marker consisted of two polymorphic alleles, as observed with the SSR marker NAU2251 [27]. One polymorphic allele identified as NAU2251_165 in Hai7124 and NAU2251_170 in TM-1 was mapped to A12, while the other was mapped to D12 by NAU2251_155 in Hai7124 and NAU2251_160 in TM-1. Two types of BAC clones were identified by the SSR marker NAU2251. According to the polymorphic allele, BAC clone z84A22 (Fig. 2a, lane 3) contained the polymorphic locus NAU2251_165, and 259 L20 (Fig. 2a, lane 4) contained the polymorphic locus NAU2251_155 between TM-1 and Hai7124 identified using SSR marker NAU2251. BAC clones z84A22 and 259 L20 were therefore mapped to A12 and D12. Probe FISHing confirmed that BAC clones z84A22 and 259 L20 mapped to a pair of chromosomes (Fig. 2b). Additionally, the SSR marker produced two alleles in another case, one at a polymorphic locus, and the other at a monomorphic locus. The SSR marker NAU3441 produced two PCR fragments in both G. barbadense cv. Hai7124 and G. hirsutum acc. TM-1 (Fig. 3), one of which was co-dominant and polymorphic (NAU3441_180 in Hai7124 and NAU3441_170 in TM-1) and mapped to 12D [33]. The other NAU3441_160 fragment was monomorphic between Hai7124 and TM-1 and could not be mapped as it is located on its homoeologous chromosome. BACFISHing confirmed that one locus originated from A12 or D12 and the other locus originated from its homoeologous chromosome [15]. The corresponding BACs were therefore distinguished using the SSR markers as described above. In total, 775 BACs were identified, with

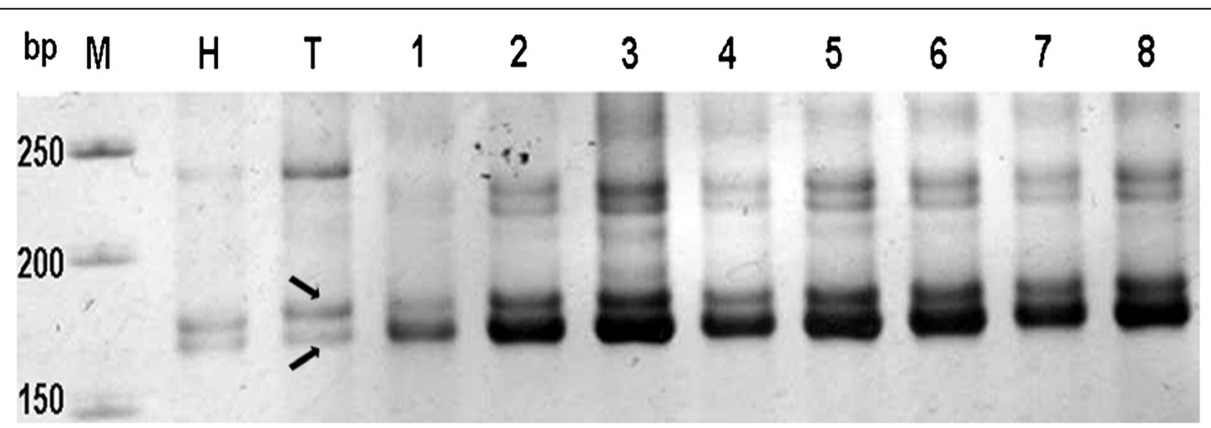

Fig. 1 Identification of BAC library with dPL0240. The dPL0240-based PCR product of parents and positive BAC clones is denoted by the single arrow. The arrow denotes a positive BAC clone that is screened from TM-1 BAC library. The positive BAC clone has the same band as the parent(TM-1); M:Marker,H:Hai7124,T:TM-1,1-8: Positive BAC clone 


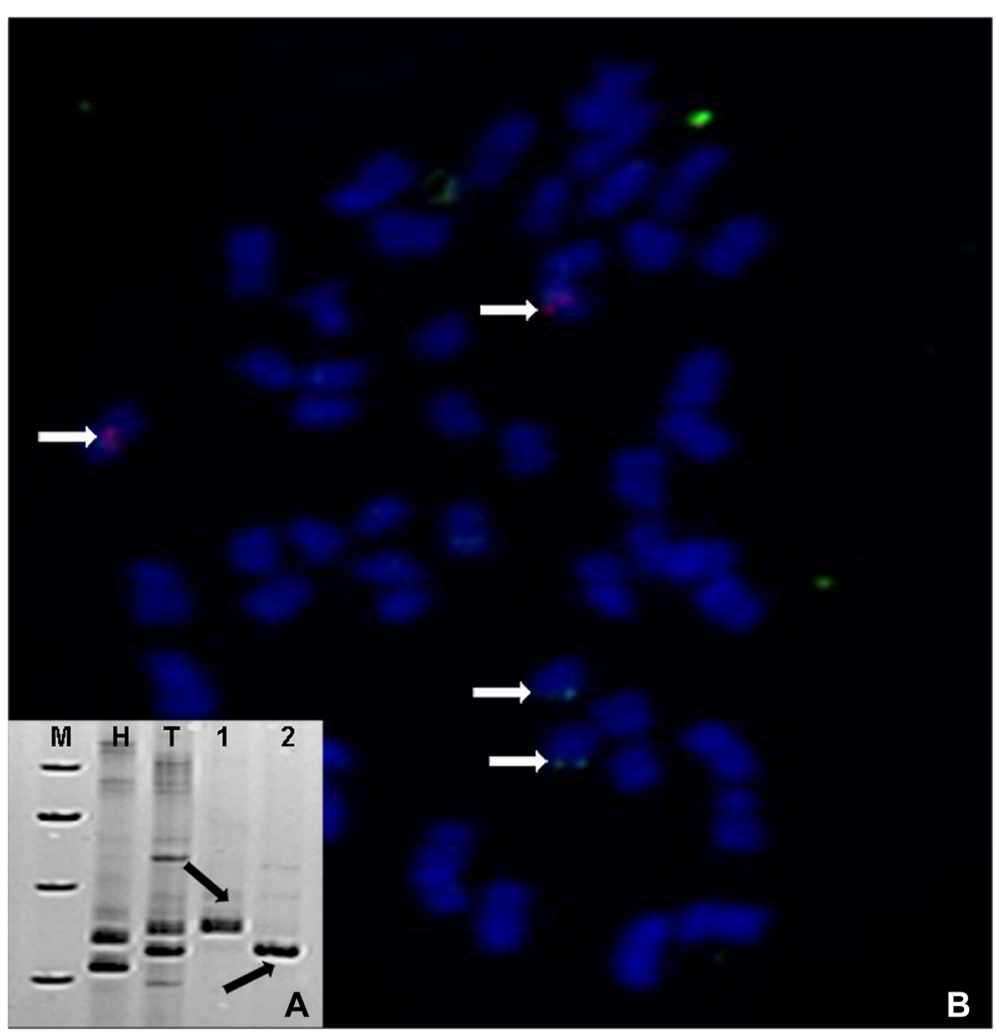

Fig. 2 Identification of screening BAC library with NAU2251 and the loacaction of postive BAC clone with BAC-FISH. a Identification of the BAC clone Z84A22 (lane 3) containing the polymorphic locus of NAU2251-165, and 259 L20 (lane 4) containing the polymorphic locus of NAU2251-155 between TM-1 and Hai7124 by SSR marker NAU2251. Lanes 1-4 were Hai7124, TM-1, Z84A22, 259 L20 respectively. b FISH image showed that the signals of the polymorphic allele BAC $259 \mathrm{~L} 20$ (green signals, arrows) and the signals of polymorphic allele BAC z84A22 (red signals, arrows) were located on A12 and D12 homoeologous chromosomes. M: Marker, H: Hai7124, T: TM-1,1: Z84A22,2: 259 L20

387 from chromosome A12 and 388 from chromosome D12. These BAC sets were used for fingerprinting.

\section{Adjustment of FPC parameters and BAC fingerprinting} BACs were digested with HindIII, and all 775 clones were fingerprinted using an agarose gel-based restriction fingerprinting method. Bands ranged in size from $1000 \mathrm{bp}$ to $21,226 \mathrm{bp}$, and ranged from $10-20$ pieces. A representative DNA fingerprinting gel is shown (Fig. 4). Bands were imaged using the Image3.10b software and saved as a bands file. A total of 387 clones from A12 produced 5372 fingerprint bands

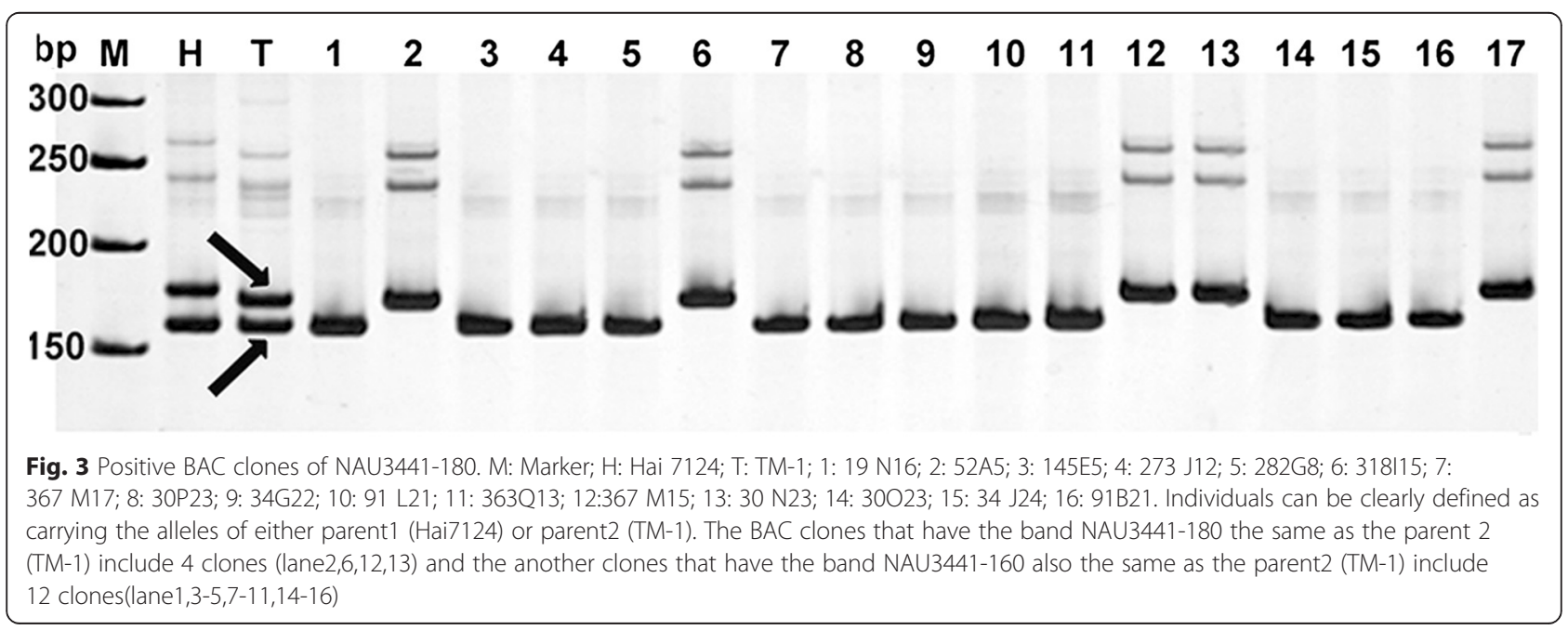




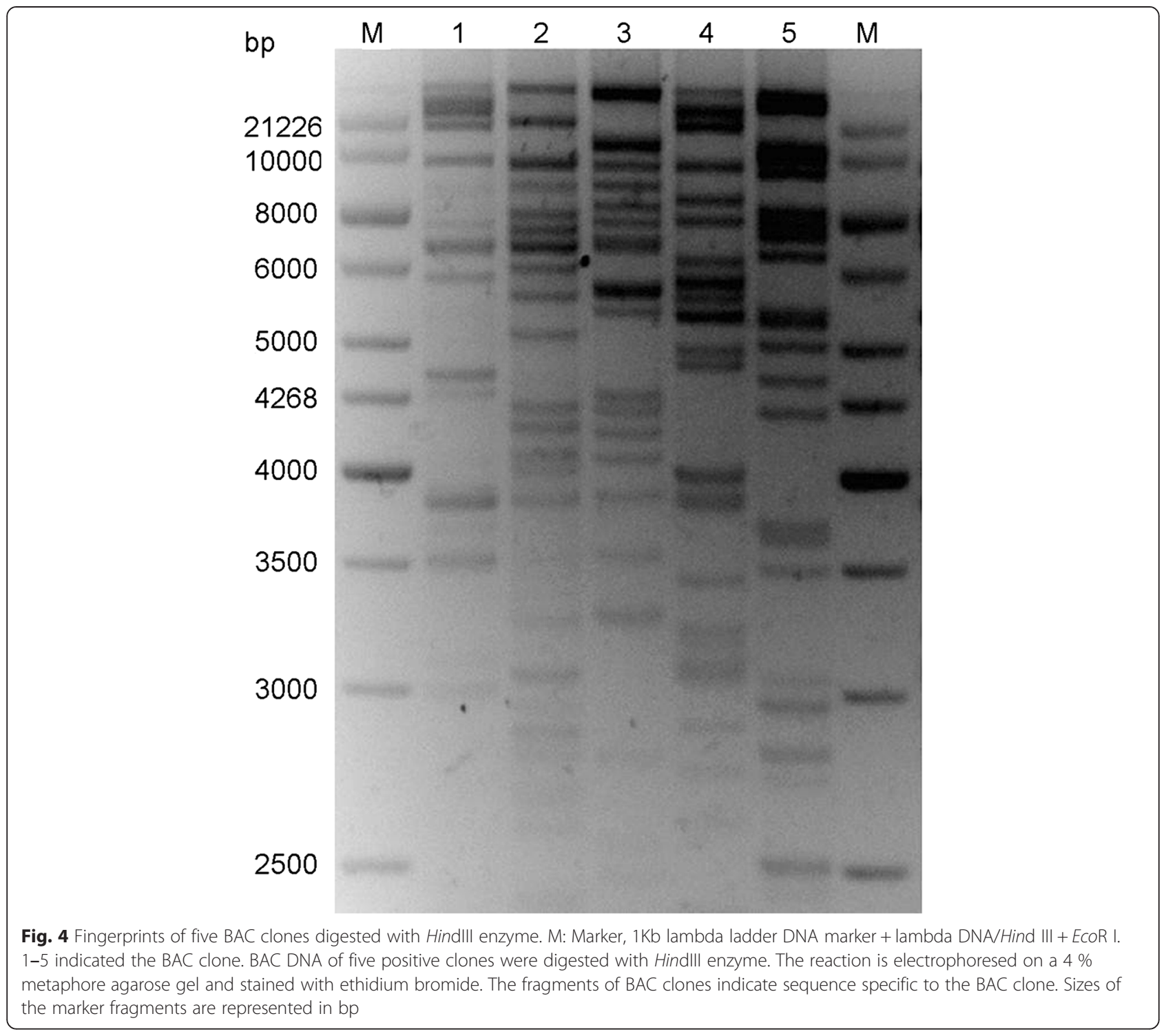

and 388 clones from D12 produced 4843 fingerprint bands.

Tolerance and cutoff values are two important parameters in the FPC software, and it is important to set these parameters appropriately for construction of a physical map to avoid false positives. To achieve the best contig map, BACs which had seven pairs of SSR markers located on A12 and D12 were chosen for contig analysis (Table 1). Firstly, the default values of tolerance (7) and cutoff (1e-10) were tested, and all BAC clones from A12 and D12 identified as described above were combined to construct contigs. Two types of contig were generated (Fig. 5). The first type resulted from four group markers (NAU2356, NAU3441, NAU2715, NAU1237) and consisted of BAC clones from A12 and D12 (Fig. 5, solid line), which were combined to produce a single contig. The second type resulted from three group markers
(NAU2251, NAU3293 and NAU1151) and consisted of BAC clones from A12 and D12 (Fig. 5, dotted line). These contigs were constructed separately for A12 and D12. It was clear that construction of contig maps for BACs from A12 and D12 separately to distinguish between the A and D subgenomes would be difficult with the default tolerance and cutoff values. We therefore adjusted these parameters in FPCV9.3 to generate a higher quality contig map for the analysis of enzyme fingerprinting. The tolerance value was set between 5 and 10 , and the cutoff value was set between 1e-01 and 1e-12.

All BAC clones identified by NAU1151 produced two types of contigs. With a tolerance value between 5 and 10 and a cutoff value of $1 \mathrm{e}-09$, the BACs from A12 and D12 combined to produce a single contig (Fig. 6a). However, when the cutoff value was increased from $1 \mathrm{e}-10$ to $1 \mathrm{e}-12$, $\mathrm{BAC}$ clones from $\mathrm{A} 12$ and $\mathrm{D} 12$ produce two contigs. 
Table 1 BACs identified by seven pairs of SSR markers

\begin{tabular}{|c|c|c|}
\hline Marker & $\begin{array}{l}\text { Molecular } \\
\text { weight }\end{array}$ & Clone ID \\
\hline NAU2715(A12) & 180 & $283 \mid 16,10 \mathrm{G} 14,53 \mathrm{C} 7$ \\
\hline NAU2715(D12) & 250 & $181 \mathrm{G} 1,255 \mathrm{~F} 14,79 \mathrm{G} 11,89 \mathrm{P} 7$ \\
\hline NAU3441(A12) & 230 & 30 N23,367 M15,318115 \\
\hline NAU3441(D12) & 180 & $\begin{array}{l}367 \text { M17,91 L21,19 N16,34 J24,34G22, } \\
273 \mathrm{J12}, 282 \mathrm{G} 8,91 \mathrm{~B} 21,363 \mathrm{Q} 13\end{array}$ \\
\hline NAU1151(A12) & 160 & $194 \mathrm{H} 15,031 \mathrm{~K} 3$ \\
\hline NAU1151(D12) & 170 & 29B22, 08119 \\
\hline NAU3293(A12) & 180 & $96 \mathrm{~K} 17$ \\
\hline NAU3293(D12) & 150 & 225C9, 206 L15, 254 L5,192 F22 \\
\hline NAU2251(A12) & 165 & $64 \mathrm{E} 13,79 \mathrm{~A} 4, \mathrm{z} 84 \mathrm{~A} 22$ \\
\hline NAU2251(D12) & 155 & 259 L20 \\
\hline NAU1237(A12) & 255 & 051 L3, 82D24, $081 \mathrm{~K} 8$ \\
\hline NAU1237(D12) & 150 & 215O23, 266E15, 45H11, 115D23 \\
\hline NAU2356(A12) & 150 & $\begin{array}{l}\text { 14P1, 22Q13, } 69 \mathrm{~K} 1,86 \mathrm{E} 19,269 \mathrm{~B} 19,271 \mathrm{D} 5, \\
\text { 294Q20, 30121, 68015, 084P1, 74E18, 74 F9, } \\
96120\end{array}$ \\
\hline NAU2356(D12) & 170 & $075 \mathrm{~A} 11,321 \mathrm{~J} 20,102 \mathrm{~N} 2$ \\
\hline
\end{tabular}

For NAU2715, with a tolerance value of 5 and a cutoff value of $1 \mathrm{e}-12$, combining all BAC clones produced two contigs from A12 and D12, respectively (Fig. 6b). However when the tolerance value was increased to 7 and the cutoff value was $1 \mathrm{e}-12$, BAC clones produced two contigs from A12 and D12, but BAC 81G1 appeared in D12 (Fig. 6b). Increasing the tolerance value to 10 and produced a one single contig from D12 (Fig. 6b).

Combining all BAC clones from NAU1237 produced two contigs with a tolerance value of 7 and a cutoff value of 1e-10, which could not distinguish contigs between chromosomes A12 and D12 (Fig. 6c). Two contigs for A12 and D12 were produced if the tolerance value was 5 and the cutoff value was $1 \mathrm{e}-11$ (Fig. 6c). When the cutoff value was $1 \mathrm{e}-11$ and the tolerance value was increased from 6 to 8 , or when the tolerance value was 5 and the cutoff value was increased to $1 \mathrm{e}-12$, this also produced separate contigs from A12 and D12, but BAC 045H11 disappeared from chromosome D12. When the tolerance value was 7 or 8 and the cutoff value was increased to $1 \mathrm{e}-12$, the BAC clones generated separate contigs from A12 and D12, but BAC clone 266E15 disappeared from chromosome D12.

Combining all BAC clones from NAU2356 produced a single contig when the tolerance value was 7 and the cutoff value was $1 \mathrm{e}-10$, which could not distinguish between A12 and D12 (Fig. 6d). With a tolerance value of 7 and a cutoff value of $1 \mathrm{e}-12$, all BAC clones from

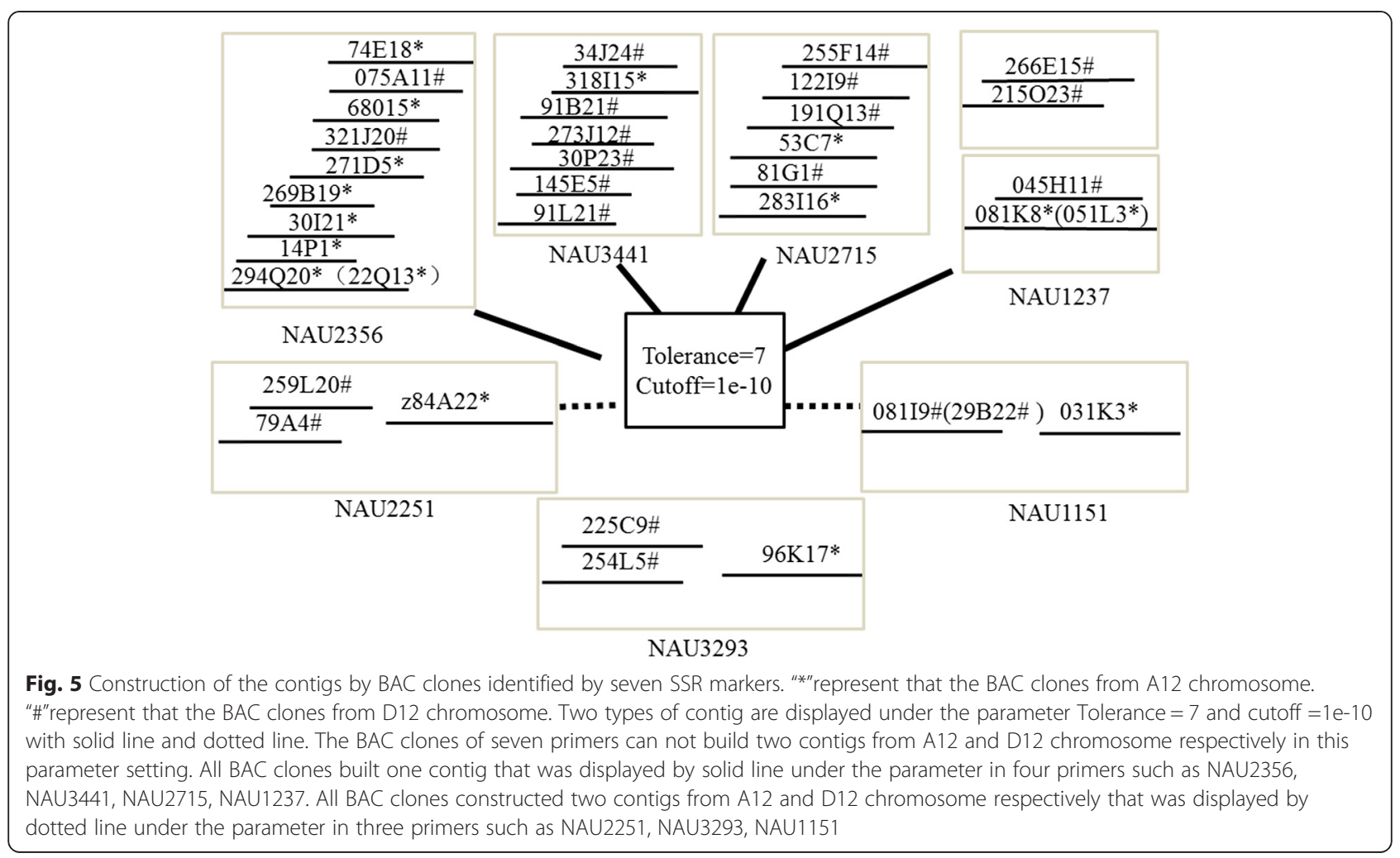




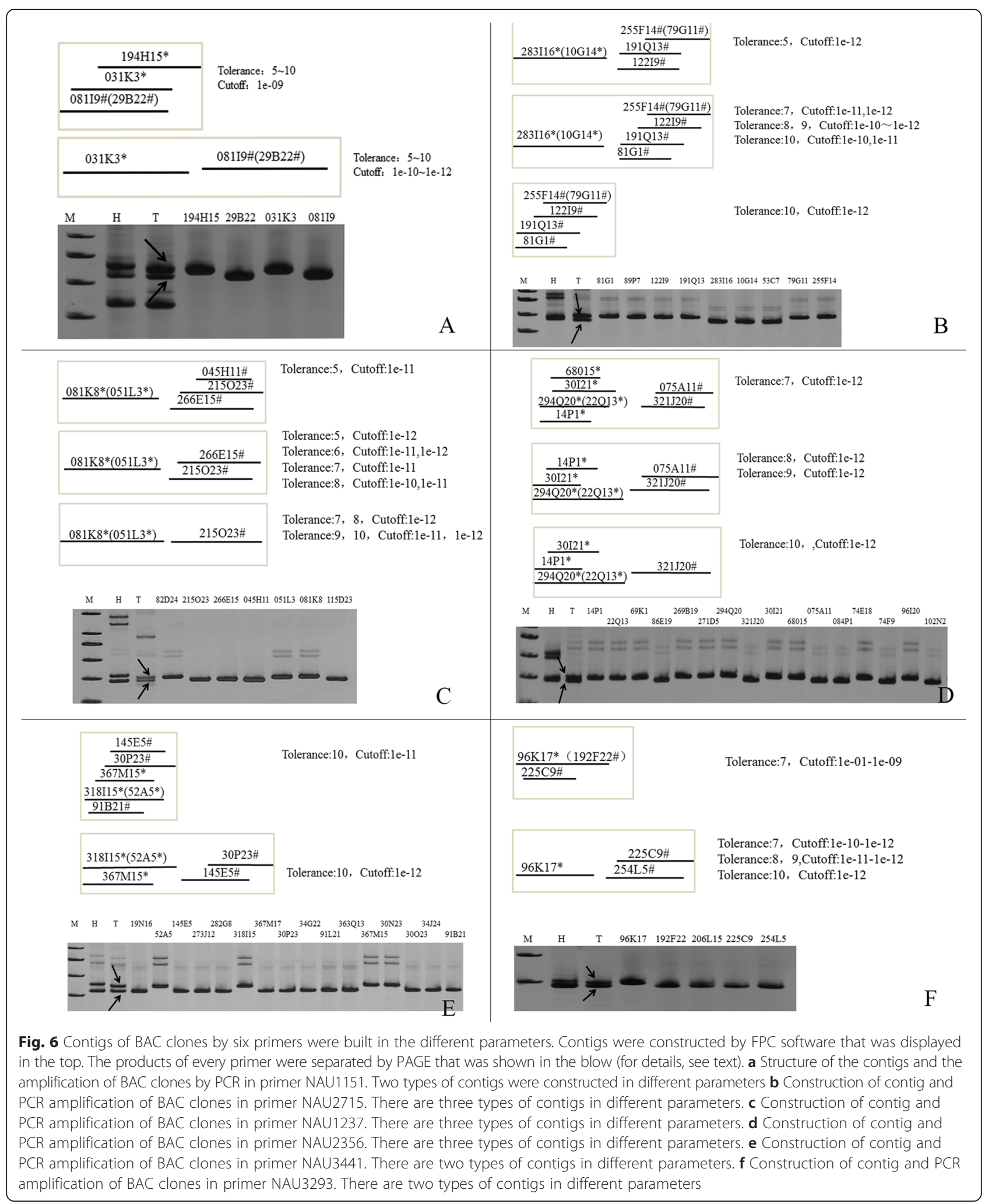


NAU2356 produced two separate contigs for A12 and D12 (Fig. 6d). Increasing the tolerance value from 8 and 9 and setting the cutoff value at $1 \mathrm{e}-12$ produced the contig from A12 and D12, respectively, but BAC 68015 disappeared from A12 (Fig. 6d). Increasing the tolerance value to 10 and setting the cutoff value at $1 \mathrm{e}-12$ produced the contig from A12 and D12, respectively, but BAC 68015 and 075A11 disappeared from contigs (Fig. 6d).

Combining BAC clones from NAU3441 produced one contig when the parameters were set at the lower end of the range (Fig. 6e). However if the tolerance value was increased to 10 and the cutoff value was set at $1 \mathrm{e}-11$, a single contig was generated (Fig. 6e), whereas with a tolerance value of 10 and a cutoff value of 1e-12, two separate contigs for A12 and D12 resulted (Fig. 6e).

Combining BAC clones from NAU3293 produced a single contig with a tolerance value of 7 and a cutoff value of 1e-01 to 1e-09 (Fig. 6f). However, increasing the cutoff value from $1 \mathrm{e}-10$ to $1 \mathrm{e}-12$ produced two contigs from A12 and D12, respectively (Fig. 6f). A tolerance value of 10 and a cutoff value of $1 \mathrm{e}-12$ were therefore optimal.

Fingerprints of all 755 BACs were assembled using the optimized parameters (tolerance value 10, cutoff value 1e-12). A total of 77 contigs from A12 were generated, ranging in size from $53 \mathrm{~kb}$ to $155 \mathrm{~kb}$, whereas 82 individual contigs were produced for D12 ranging from $61 \mathrm{~kb}$ to $139 \mathrm{~kb}$ (Fig. 7).

\section{Discussion}

\section{Using BACs as a tool for constructing contig maps}

Various strategies have been developed for constructing physical maps, and BAC clones are especially important for both map-based cloning and sequencing of large complex genomes containing abundant repetitive sequences and highly homologous segments. How to effectively distinguish between the homologous segments

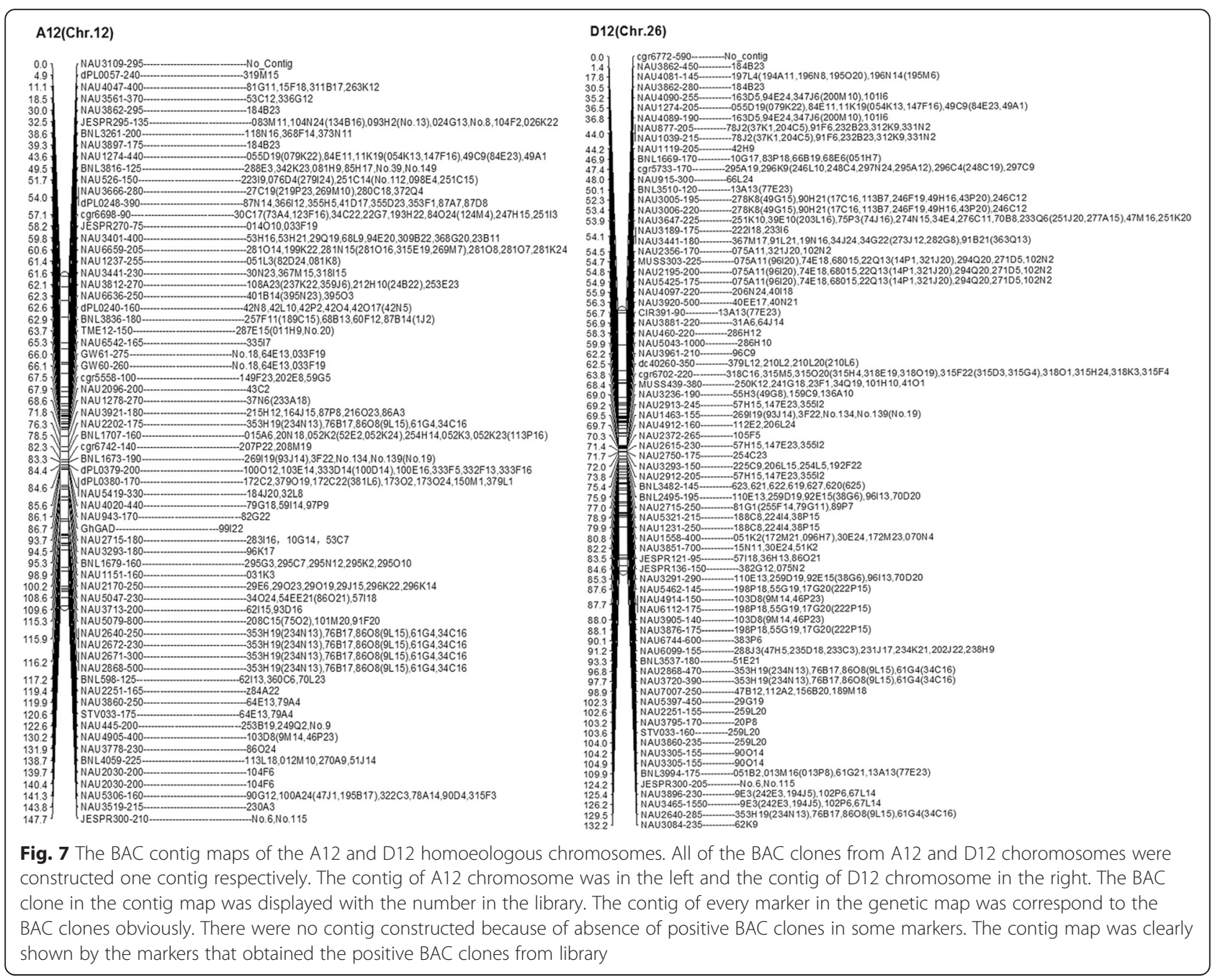


of the tetraploid cotton genome, verifying the quality of the physical map is crucial. To avoid interference from sequence similarity, BACs identified by SSR markers based on the linkage map of $12 \mathrm{~A}$ and $12 \mathrm{D}$ were used for assembly in this study. Full use was made of SSR markers from high density genetic mapping to screen the BAC library and to distinguish between the A and D subgenomes. BAC clones obtained by PCR were located to A12 or D12 homoeologous chromosomes according to the published linkage map [27]. Although BAC library screening was time consuming initially, the resulting BAC contigs were accurate and this simplified the analysis later. The first integrated cytogenetic and linkage maps of homoeologous chromosomes 12A and 12D indicate that the orders of most genetic markers tested are colinear with corresponding BAC FISH signals. Although the orders and positions of polymorphic and monomorphic BACs on chromosome 12A and 12D were concordant with marker positions in the corresponding linkage map, the detailed chromosomal view of genome size variation between homoeologous chromosomes 12A and $12 \mathrm{D}$ was provided that show 1.3-fold size variation [35]. Furthermore, upon integrating with molecular markers, the BAC contig map of A12 and D12 will facilitate map-based cloning of QTLs or genes associated with important agronomic traits and marker-assisted selection, as well as comparative studies for analysis of the evolution of cotton genomes using sequencing of homologous segments.

\section{Improving contig map quality}

The quality of the BAC library DNA is important for sharp, clear fingerprinting maps, and several steps in the preparation of BAC DNA are critical to the success of the process. Due to the low copy number, at least $3 \mathrm{ml}$ of cell culture should be used for DNA preparation, and both cell growth and BAC production should be adequate. The cell pellet should be thoroughly suspended and cell lysis should proceed for less than 5 min. For construction of contigs, the tolerance and cutoff parameters were highly influential. We tested cutoff values from $1 \mathrm{e}-01$ to $1 \mathrm{e}-12$ and tolerance values from 5 to 10 , which span the range used widely for agarose-based enzyme fingerprinting. A tolerance value of between 3 and 5 and a cutoff value between $1 \mathrm{e}-30$ and $1 \mathrm{e}-50$ are generally used in HICF fingerprint analysis [36]. A tolerance value of 4 and a cutoff value from $1 \mathrm{e}-20$ to $1 \mathrm{e}-04$ were selected for the BIBAC contig map, which contained at least five shared clones [37], and a tolerance value of 8 and a cutoff value of 1e-10 were used in the Gossypium raimondii D-genome physical map [38]. In this study, we adjusted the parameters in the FPCV9.3 software to improve the quality of the contig map for analysis by enzyme fingerprinting using homologous
BACs. A tolerance value of 10 and a cutoff value of $1 \mathrm{e}-$ 12 were found to be the optimal parameters.

\section{Conclusions}

In this study, we successfully constructed a BAC contig map of the homoeologous chromosome 12 of G. hirsutum TM-1. The BAC-based method was an effective strategy for construction of the cotton physical map, but further work is necessary to improve the contig map. Even so, this work introduces a novel method for combined analysis of homoeologous chromosomes, and could provide an important framework for sequencing of the cotton genome. This work could also facilitate research into the evolution of the cotton genome through sequencing of homologous segments and comparison of genome sequences with BAC sequences. This could also generate information on the genome formation and evolutionary processes involved in cotton polyploidization.

\section{Methods}

\section{Source BAC library and BAC library screening}

A BAC library constructed from G. hirsutum cv. Texas Marker-1 (TM-1) was used in this study [27]. The library was constructed from cotton DNA partially digested with Hind III and incorporated into the BAC vector pIndigBAC-5. The library consists of 147,456 clones with an average insert size of $122.8 \mathrm{~kb}$ that ranges from 97 to $240 \mathrm{~kb}$. Approximately $96 \%$ of the clones contain inserts over $100 \mathrm{~kb}$, therefore this library represents 7.4 haploid genome equivalents in theory, based on an $\mathrm{AD}$ genome size of $2425 \mathrm{Mb}$. Clones were stored in 384-well plates and arrayed into multiplex pools for rapid and reliable library screening. BAC screening was carried using four-round PCR using SSR markers selected from the A12 and D12 high-density genetic maps derived from populations of the tetraploid Gossypium species.

\section{BAC-DNA isolation and fingerprinting}

BAC clones were inoculated into 96 -well $2.2 \mathrm{~mL}$ plates, and each well contained $1.5 \mathrm{~mL}$ of $2 \mathrm{xYT}$ medium $(12.5 \mu \mathrm{g} / \mathrm{mL} \mathrm{CM})$. Plates were covered with sealing film and incubated at $37{ }^{\circ} \mathrm{C}$ for $20-24 \mathrm{~h}$ on a shaker. BAC DNA was isolated using standard alkaline lysis [39], digested with HindIII, and subjected to $1 \%$ agarose gel electrophoresis at $40 \mathrm{~V}$ for $16 \mathrm{~h}$. Restriction fragment identification was performed using IMAGE 3.10b software [40] with extensive manual editing. Fragments ranging from 53 to 155 bases were used for contig assembly. Bands derived from the BAC vector (pIndigBAC-5) and BACs containing less than five bands were manually deleted from the image files. 


\section{BAC contigs assembly}

The computer program FPC V9.3 (http://www.agcol.arizona.edu/software/fpc/) was used to assemble the physical map contigs from the BAC fingerprints. A series of tests were conducted in which fingerprints of a set of overlapping clones were compared using different tolerance values (from 5 to 10 ) and cutoff values (from 1e-01 to $1 \mathrm{e}$ 12). Based on these results, a tolerance of 10 and a primary cutoff of 1e-12 were selected for contig assembly.

\section{Competing interests}

The authors declare that they have no competing interests.

\section{Authors' contributions}

$\mathrm{YHL}$ and $\mathrm{YH}$ performed all the experiments and drafted the manuscript. DM, WHL and YDL analyzed the data. YH, WZG and TZZ conceived the study, participated in its design and coordination, drafted and revised the manuscript. All authors read and approved the final manuscript.

\section{Acknowledgements}

This study was financially supported in part by grants from the National Natural Science Foundation (31201250), the Natural Science Foundation in Jiangsu Province (BK2010441), the Priority Academic Program Development of Jiangsu Higher Education Institutions and 111 Project.

Received: 24 April 2015 Accepted: 9 July 2015

Published online: 28 July 2015

\section{References}

1. Shizuya H, Birren B, Kim UJ, Mancino V, Slepak T, Tachiiri Y, et al. Cloning and stable maintenance of 300-kilobase-pair fragments of human DNA in escherichia-coli using an F-factor-based vector. Proc Natl Acad Sci U S A. 1992;89(18):8794-7.

2. Woo SS, Jiang J, Gill BS, Paterson AH, Wing RA. Construction and characterization of a bacterial artificial chromosome library of Sorghum bicolor. Nucleic Acids Res. 1994:22(23):4922-31.

3. Staden R. A mew computer method for the storage and manipulation of DNA gel reading data. Nucleic Acids Res. 1980;8(16):3673-94.

4. Coulson A, Sulston J, Brenner S, Karn J. Toward a physical map of the genome of the nematode Caenorhabditis elegans. Proc Natl Acad Sci U S A. 1986:83(20):7821-5.

5. Frelichowski Jr JE, Palmer MB, Main D, Tomkins JP, Cantrell RG, Stelly DM Cotton genome mapping with new microsatellites from Acala 'Maxxa' BAC-ends. Mol Gen Genet. 2006;275:479-91.

6. Wang XF, Jun MA, Ma ZY, Zhang GY, Zheng YM. BAC library construction and characterization of Suyuan7235, a cotton germplasm with high fiber strength. Cotton Sci. 2006;18:200-3 (in Chinese with an English abstract).

7. Zheng YM, Wang XF, Zhang GY, Li XH, Ma ZY. BAC library construction of Zhongmiansuo 12 with high yield, high quality and disease resistance. J Hebei Agric Univ. 2004:27:17-20 (in Chinese with an English abstract).

8. Yin JM, Guo WZ, Yang LM, Liu LW, Zhang TZ. Physical mapping of the $R f_{7}$ fertility-restoring gene to a $100 \mathrm{~kb}$ region in cotton. Theor Appl Genet. 2006;112(7):1318-25

9. Wang XF, Ma J, Wang WS, Zheng YM, Zhang GY, Liu CJ. Construction and characterization of the first bacterial artificial chromosome library for the cotton species Gossypium barbadense L. Genome. 2006:49:1393-8.

10. Kim HJ, Triplett BA. Cotton fiber growth in planta and in vitro. Models for plant cell elongation and cell wall biogenesis. Plant Physiol. 2001:127(4):1361-6.

11. Fryxell P. A revised taxonomic interpretation of Gossypium L. (Malvaceae). Rheedea. 1992:2:108-65.

12. Wendel JF. New World tetraploid cottons contain Old World cytoplasm. Proc Natl Acad Sci U S A. 1989:86(11):4132-6.

13. Seelanan T, Schnabel A, Wendel JF. Congruence and consensus in the cotton tribe (Malvaceae). Syst Bot. 1997:22(2):259-90.

14. Cronn RC, Small RL, Haselkorn T, Wendel JF. Rapid diversification of the cotton genus (Gossypium: Malvaceae) revealed by analysis of sixteen nuclear and chloroplast genes. Am J Bot. 2002;89(4):707-25.
15. Wang K, Guo WZ, Zhang TZ. Detection and mapping of homologous and homoeologous segments in homoeologous groups of allotetraploid cotton by BAC-FISH. BMC Genomics. 2007;8:178.

16. Rong JK, Abbey C, Bowers JE, Brubaker CL, Chang C, Chee PW, et al. A 3347-locus genetic recombination map of sequence-tagged sites reveals features of genome organization, transmission and evolution of cotton (Gossypium). Genetics. 2004;166(1):389-417.

17. Nguyen TB, Giband M, Brottier P, Risterucci AM, Lacape JM. Wide coverage of the tetraploid cotton genome using newly developed microsatellite markers. Theor Appl Genet. 2004;109(1):167-75.

18. Wang K, Song XL, Han ZG, Guo WZ, Yu JZ, Sun J, et al. Complete assignment of the chromosomes of Gossypium hirsutum L. by translocation and fluorescence in situ hybridization mapping. Theor Appl Genet. 2006:113(1):73-80.

19. Song L, Guo WZ, Qin HD, Ding YZ, Zhang TZ. Genetic analysis and molecular validation of chromosome assignment for fuzzless genes $N_{1}$ and $n_{2}$ in cotton. J Nanjing Agric Univ. 2010;33(1):21-6 (In Chinese).

20. Stelly DM. Localization of the Le 2 Locus of Cotton (Gossypium-Hirsutum L). J Hered. 1990;81:193-7.

21. Samora P, Stelly D, Kohel R. Localization and mapping of the $L e_{7}$, and $G l_{2}$ loci of cotton (Gossypium hirsutum L.). J Hered. 1994;85:152-7.

22. Kohel RJ, Stelly DM, Yu J. Tests of six cotton (Gossypium hirsutum L.) mutants for association with aneuploids. J Hered. 2002;93:130-2.

23. Endrizzi JE, Turcotte EL, Kohel RJ. Genetics, cytogenetics and evolution of Gossypium. Adv Genet. 1985:23:271-5.

24. Rong J, Pierce GJ, Waghmare VN, Rogers CJ, Desai A, Chee PW, et al. Genetic mapping and comparative analysis of seven mutants related to seed fiber development in cotton. Theor Appl Genet. 2005;111(6):1137-46.

25. Dong CG, Ding YZ, Guo WZ, Zhang TZ. Fine mapping of the dominant glandless Gene G/2 in Sea-island cotton (Gossypium barbadense L.). Chin Sci Bull. 2007;52(22):3105-9.

26. Chen D, Ding Y, Guo W, Zhang T. Molecular mapping of genic male-sterile genes $m s_{15}, m s_{5}$ and $m s_{6}$ in tetraploid cotton. Plant Breed. 2009;128(2):193-8

27. Hu Y, Guo WZ, Zhang TZ. Construction of a bacterial artificial chromosome library of TM-1, a standard line for genetics and genomics in Upland cotton. J Integr Plant Biol. 2009;51(1):107-12.

28. Xu Z, Kohel RJ, Song G, Cho J, Yu J, Yu S, et al. An integrated genetic and physical map of homoeologous chromosomes 12 and 26 in Upland cotton (G. hirsutum L.). BMC Genomics. 2008;9:108.

29. Han ZG, Guo WZ, Song XL, Zhang TZ. Genetic mapping of EST-derived microsatellites from the diploid Gossypium arboreum in allotetraploid cotton. Mol Genet Genomics. 2004;272(3):308-27.

30. Song XL, Wang K, Guo WZ, Zhang J, Zhang TZ. A comparison of genetic maps constructed from haploid and $\mathrm{BC}_{1}$ mapping populations from the same crossing between Gossypium hirsutum L. and Gossypium barbadense L. Genome. 2005:48(3):378-90.

31. Han ZG, Wang CB, Song XL, Guo WZ, Gou JY, Li CH, et al. Characteristics, development and mapping of Gossypium hirsutum derived EST-SSRs in allotetraploid cotton. Theor Appl Genet. 2006;112(3):430-9.

32. Guo WZ, Cai CP, Wang CB, Han ZG, Song XL, Wang K, et al. A microsatellite-based, gene-rich linkage map reveals genome structure, function and evolution in gossypium. Genetics. 2007;176(1):527-41.

33. Guo WZ, Cai C, Wang C, Zhao L, Wang L, Zhang TZ. A preliminary analysis of genome structure and composition in Gossypium hirsutum. BMC Genomics. 2008;9:314.

34. Zhao L, YD L, Cai CP, Tong XC, Chen XD, Zhang W, et al. Toward allotetraploid cotton genome assembly: integration of a high-density molecular genetic linkage map with dna sequence information. BMC Genomics. 2012;13:539.

35. Wang K, Guo W, Yang Z, Hu Y, Zhang W, Zhou B, et al. Structure and size variations between 12A and 12D homoeologous chromosomes based on high-resolution cytogenetic map in allotetraploid cotton. Chromosoma. 2010:119:255-66.

36. Luo MC, Thomas C, You FM, Hsiao J, Shu OY, Buell CR, et al. Highthroughput fingerprinting of bacterial artificial chromosomes using the SNaPshot labeling kit and sizing of restriction fragments by capillary electrophoresis. Genomics. 2003;82(3):378-89.

37. Zhang M, Zhang Y, Huang JJ, Zhang X, Lee MK, Stelly DM, et al. Genome physical mapping of polyploids: a BIBAC physical map of cultivated tetraploid cotton, Gossypium hirsutum L. Plos One. 2012;7(3):e33644. 
38. Lin L, Pierce GJ, Bowers JE, Estill JC, Compton RO, Rainville LK, et al. A draft physical map of a D-genome cotton species (Gossypium raimondii). BMC Genomics. 2010;11:395.

39. Sambrook J, Fritsch EF, Maniatis T. Molecular Cloning: A Laboratory Manual. 2nd ed. New York: Cold Spring Harbor Laboratory Press; 1989.

40. Sulston J, Mallett F, Durvin R, Horsnell T. Image analysis of restriction enzyme fingerprint audioradiograms. Comput Appl Biosci. 1989;5:101-6.

Submit your next manuscript to BioMed Central and take full advantage of:

- Convenient online submission

- Thorough peer review

- No space constraints or color figure charges

- Immediate publication on acceptance

- Inclusion in PubMed, CAS, Scopus and Google Scholar

- Research which is freely available for redistribution 